\title{
Thermally activated escape rate for a Brownian particle in a tilted periodic potential for all values of the dissipation
}

\author{
W. T. Coffey \\ Department of Electronic and Electrical Engineering, Trinity College, Dublin 2, Ireland \\ Yu. P. Kalmykov \\ Laboratoire de Mathématiques et Physique des Systèmes, Université de Perpignan, 52, Avenue de Paul Alduy, \\ 66860 Perpignan Cedex, France \\ S. V. Titov \\ Institute of Radio Engineering and Electronics of the Russian Academy of Sciences, Vvedenskii Square 1, Fryazino, Moscow Region, \\ 141190, Russian Federation \\ B. P. Mulligan \\ Department of Electronic and Electrical Engineering, Trinity College, Dublin 2, Ireland
}

(Received 8 February 2006; published 7 June 2006)

\begin{abstract}
The translational Brownian motion of a particle in a tilted washboard potential is considered. The dynamic structure factor and longest relaxation time are evaluated from the solution of the governing Langevin equation by using the matrix continued fraction method. The longest relaxation time is compared with the Kramers theory of the escape rate of a Brownian particle from a potential well as extended to the Kramers turnover region by Mel'nikov [Physics Reports 209, 1 (1991)]. It is shown that in the low temperature limit, the universal Mel'nikov expression for the escape rate provides a good estimate of the longest relaxation time for all values of dissipation including the very low damping (VLD), very high damping (VHD), and turnover regimes. For low barriers (where the Mel'nikov method is not applicable) and zero tilt, analytic equations for the relaxation times in the VLD and VHD limits are derived.
\end{abstract}

DOI: 10.1103/PhysRevE.73.061101

PACS number(s): 05.40.Jc, 05.10.Gg

\section{INTRODUCTION}

The translational Brownian motion in the tilted washboard potential

$$
V(z)=-V_{0} \cos (2 \pi z / a)-F z+\text { const },
$$

where $z$ is the coordinate and $a$ is a characteristic length with the constant field driving potential $F z$ superimposed on the periodic potential $-V_{0} \cos (2 \pi z / a)$, arises in a number of important physical applications involving noise and relaxation processes in phase-locked loops. We mention the currentvoltage characteristics of the Josephson junction $[1,2]$, mobility of superionic conductors [3], a laser with injected signal [4], phase-locking techniques in radio engineering [5], dielectric relaxation (when $F=0$ ) of molecular crystals [6], the dynamics of a charged density wave condensate in an electric field [7], ring-laser gyroscopes [8], stochastic resonance $[9,10]$, etc. A comprehensive discussion of the model is given in Refs. $[1,11,12]$.

One of the most important characteristics associated with the Brownian motion in any multiwell and single-well potential is the friction and temperature dependence of the greatest (overbarrier) relaxation time which is essentially the inverse of the smallest nonvanishing eigenvalue $\lambda_{1}$ of the characteristic equation or secular determinant of the relevant dynamical system. In other words, $\lambda_{1}^{-1}$ is the lifetime of the longest lived relaxation mode of the system. The greatest relaxation time may also be obtained by calculating the mean first passage times from each of the wells of the potential [13]. As far as the calculation of $\lambda_{1}$ is concerned, one may expand the solution of the associated probability density diffusion equation (usually the specialized form of the Fokker-Planck equation applicable to separable and additive Hamiltonians comprising the sum of the potential and kinetic energies known as the Klein-Kramers equation) in Fourier series in the position and velocity variables [11]. Alternatively, the secular equation may be generated by averaging the appropriate Langevin equation over its realizations in phase space yielding the hierarchy of differential-recurrence equations governing the decay functions of the system [12]. In each of the two methods, the secular determinant results from truncation of the set of differential-recurrence relations at a number ensuring convergence of the resulting set of simultaneous ordinary differential equations. Thus, $\lambda_{1}$ is not in general available in closed form as it is always rendered as the smallest root of a high order polynomial equation. Hence, it is difficult to compare $\lambda_{1}$ so determined with experimental observations of the greatest relaxation time or the relaxation rate. Fortunately (noting that $\lambda_{1}$ for sufficiently high barriers has exponential dependence on the barrier height), a way of overcoming this difficulty is to utilize an ingenious method originally proposed by Kramers [14] in connection with thermally activated escape of particles out of a potential well.

Kramers [14] (using Einstein's theory of the Brownian motion as extended both to include the inertia of the Brownian particles and a potential well) evaluated the prefactor $A$ (which is a function of both the dissipative coupling to the bath and the potential shape) in an Arrhenius-like equation 
for the escape rate $\Gamma$ over the potential barrier $\Delta V$ (reaction velocity in the case of chemical reactions), viz.,

$$
\Gamma=A \frac{\omega_{a}}{2 \pi} e^{-\Delta V / k_{B} T}, \quad \Delta V \gg k_{B} T,
$$

where the attempt frequency $\omega_{a}$ is the angular frequency of a particle executing oscillatory motion at the bottom of a well (for reviews of applications of Kramers' method see Refs. $[13,15]), k_{B}$ is Boltzmann's constant, and $T$ is temperature. Due to the unique contribution of Kramers to our understanding of the role played by the dissipative coupling in the escape rate, the escape-rate problem is now commonly known as the Kramers problem [13].

Now if the escape rates for Brownian particles governed by separable and additive Hamiltonians comprising the sum of the kinetic and potential energy are calculated by the Kramers method, three regimes of damping automatically appear, viz., (i) intermediate-to-high damping (IHD), (ii) very low damping (VLD), and (iii) a turnover region. Kramers [14] obtained so-called IHD and VLD formulas for the escape rate, assuming in both cases that the energy barrier is much greater than the thermal energy so that the concept of an escape rate applies. He mentioned in his paper, however, that he could not find a general method of attack for the purpose of obtaining a formula which would be valid for any damping regime. This problem was solved nearly 50 years later by Mel'nikov and Meshkov [16,17]. They postulated from heuristic reasoning that a formula valid for all values of the damping may be given by simply multiplying the general Kramers IHD result for $\Gamma$ by using a certain bridging integral derived by them. Mel'nikov [17] further extended the bridging integral method to take into account quantum effects in a semiclassical way. Later Grabert [18] and Pollak et al. [19] presented an even more complete solution of the Kramers turnover problem showing that the Mel'nikov and Meshkov turnover formula for the escape rate can be obtained without ad hoc interpolation between the weak and strong damping regimes. We remark that the theory of Pollak et al. [19] is also applicable to an arbitrary memory friction and not only in the "white noise" (memoryless) limit. In the semiclassical limit, the latter theory was extended to the quantum regime by Rips and Pollak [20].

As far as general accuracy is concerned, the universal turnover formula of Mel'nikov and Meshkov compares favorably with escape rate calculations based on either the solution of the Klein-Kramers equation or on numerical simulations of the Brownian dynamics in various potentials (not including to date, however, the tilted cosine potential). In particular, a comparison with the numerical results for the escape out of a single well was given in Refs. [21,22]. For double-well potentials, the Mel'nikov and Meshkov formula has been tested in Refs. [23-27] for both the rotational and translational Brownian motion. Furthermore, Coffey et al. $[15,28]$ have extended the method to the magnetization relaxation of single-domain ferromagnetic particles. (The magnetic relaxation differs fundamentally from that of inertial Brownian particles because the undamped equation of motion of the magnetization of a single-domain ferromagnetic particle is the gyromagnetic equation and the Hamiltonian is nonseparable. Thus the inertia plays no role; the part played by inertia in the mechanical system is essentially mimicked in the magnetic system by the gyromagnetic term causing the coupling or "entanglement" of the transverse and longitudinal modes). The calculation of the longest relaxation time for various magnetocrystalline anisotropies has been accomplished in Refs. [29-31].

It is the purpose of this paper to apply a universal turnover formula for the escape rate to the analysis of the dynamics of a Brownian particle in the tilted periodic potential, Eq. (1). In particular application to a Josephson tunneling junction, Mel'nikov has derived such a universal equation for the longest relaxation time in Ref. [17]. In this context, we must remark that the Kramers problem in a tilted periodic potential is qualitatively different from the escape problem from a metastable well because the tilted periodic potential is multistable. The particle having escaped a particular well may again be trapped due to the thermal fluctuations in another well. Moreover, jumps of either a single lattice spacing or of many lattice spacings are possible [32]. Thus from a mathematical point of view, one has to take into account the nonperiodic solution of the Fokker-Planck equation. Hence the dynamic structure factor $\widetilde{S}(k, \omega)$ (playing an essential role in neutron and light scattering experiments) is used as described by Risken [11] generating an additional (wave number) parameter $k$ in the Fokker-Planck equation. That factor is then averaged over all possible jumps. Yet another difference from the conventional Kramers problem is that the stationary distribution is no longer the Maxwell-Boltzmann distribution. In the VHD limit, the analysis of the problem usually starts from the Smoluchowski equation by either converting the solution of that equation to a Sturm-Liouville problem or to the solution of an infinite hierarchy of linear differential-recurrence relations for statistical moments [11]. We have mentioned that a concise method of numerical treatment (in terms of infinite scalar continued fractions) of the model in the VHD limit, where the inertia of the particle may be neglected, has been suggested by Cresser et al. [33] with applications to a ring-laser gyroscope as summarized by Risken [11] (see also references cited therein). Further development of the continued fraction approach has been given by Coffey et al. $[12,34]$. In the opposite VLD limit, the calculation of the escape rate by Kramer's method was accomplished in Refs. [35,36]. A general method of solution of the problem for all values of dissipation based on a matrix continued fraction representation of the Klein-Kramers equation has been suggested by Risken [11]. This method allows one to calculate eigenvalues and eigenfunctions of the KleinKramers (Fokker-Planck) equation for the tilted periodic potential and evaluate the Fourier transforms of various correlation functions for virtually all cases. By applying this method, Ferrando et al. [32,37] have studied the onedimensional translational Brownian motion in a pure periodic potential (1) with $F=0$. These authors have evaluated numerically the escape rate $\Gamma$ from the dynamic structure factor $\widetilde{S}(k, \omega)$ and shown that the friction dependence of $\Gamma$ so obtained is in full agreement with that given by the Mel'nikov universal equation [17]. The treatment of the same one-dimensional problem and its generalization to dif- 


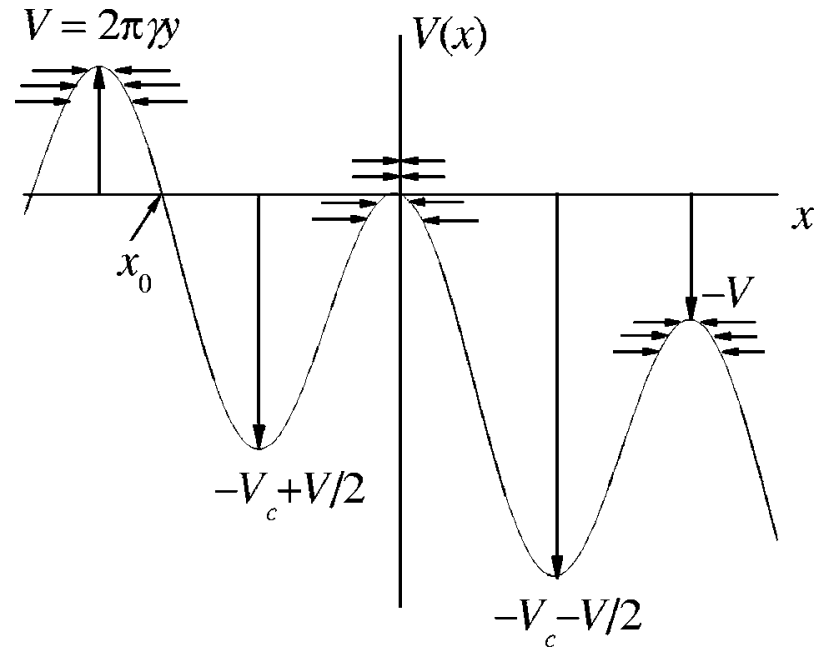

FIG. 1. Potential $V(x)=-\gamma(\cos x+y x)+C$ with $V_{c}=2 \gamma\left(\sqrt{1-y^{2}}\right.$ $+y \arcsin y)$ and $V=2 \pi \gamma y$.

fusion on a surface has been given by Pollak and collaborators in Refs. [38-40]. Neither Ferrando et al. or Pollak et al. extended their comparisons of the turnover formula with the exact solution to the tilted cosine potential, i.e., $F \neq 0$, which we reiterate is the object of the present paper. This general case differs in many respects from that considered by Ferrando et al. [32,37] as now the dynamic response strongly depends on the tilt parameter $F$. Here we present the results of a detailed comparison of the Mel'nikov turnover formula with a matrix continued fraction solution for the dynamic structure factor and longest relaxation time for the onedimensional translational Brownian motion in a tilted periodic potential, Eq. (1). In this way, the range of validity of the approximate analytic solutions for dynamic structure factor $\tilde{S}(k, w)$ and the escape rate $\Gamma$ may be ascertained. Our matrix continued fraction solution owes much to the method of Risken [11]. However, the hierarchy of differentialrecurrence equations for statistical moments [which is the basis of the evaluation of $\tilde{S}(k, \omega)]$ is derived directly from the underlying Langevin equation without recourse to the Fokker-Planck equation.

\section{BASIC EQUATIONS}

We consider the one-dimensional translational Brownian motion of a particle of mass $m$ in a tilted periodic potential, Eq. (1). On introducing the normalized coordinate $x$, time $t^{\prime}$, tilt $y$, and barrier $\gamma$ parameters as

$$
\begin{gathered}
x=\frac{2 \pi}{a} z, \quad y=\frac{a F}{2 \pi V_{0}}, \quad \gamma=\frac{V_{0}}{k_{B} T}, \quad t^{\prime}=\frac{t}{\eta}, \\
\eta=\frac{a}{2 \pi} \sqrt{\frac{m}{2 k_{B} T}},
\end{gathered}
$$

the potential Eq. (1) takes the form (see Fig. 1)

$$
V(x)=V(z) /\left(k_{B} T\right)+C^{\prime}=-\gamma(\cos x+y x)+C^{\prime},
$$

and the Langevin equation describing the dynamics of the Brownian particle becomes

$$
\ddot{x}\left(t^{\prime}\right)+\beta^{\prime} \dot{x}\left(t^{\prime}\right)+\partial_{x} V\left[x\left(t^{\prime}\right)\right] / 2=\lambda\left(t^{\prime}\right),
$$

where $C^{\prime}=-\gamma\left(\sqrt{1-y^{2}}-1+y \arcsin y\right.$ ) (this constant does not affect the equation of motion, however, it is necessary for further calculations), $\beta^{\prime}=\eta \zeta / m$ is the dimensionless friction parameter, $\zeta$ is the viscous drag coefficient, and $\lambda\left(t^{\prime}\right)$ is the white noise driving force so that

$$
\overline{\lambda\left(t^{\prime}\right)}=0, \quad \overline{\lambda\left(t_{1}^{\prime}\right) \lambda\left(t_{2}^{\prime}\right)}=\eta \beta^{\prime} \delta\left(t_{1}^{\prime}-t_{2}^{\prime}\right) .
$$

The overbar means the statistical average over an ensemble of particles which have all started at time $t^{\prime}$ with the same initial position $x\left(t^{\prime}\right)=x$ and velocity $\dot{x}\left(t^{\prime}\right)=\dot{x}$. Equation (5) is interpreted here as a stochastic differential equation of the Stratonovich type $[11,12]$. Typical examples of physical systems modeled by Brownian motion in a tilted periodic potential and described by the Langevin Eq. (5) are presented in Table I.

The corresponding Klein-Kramers (Fokker-Planck) equation for the joint probability density function $W\left(x, \dot{x}, t^{\prime}\right)$ of the phase space variables $x$ and $\dot{x}$ may be written [11]

$$
\frac{\partial W}{\partial t^{\prime}}=L_{F P} W
$$

where the Fokker-Planck operator $L_{F P}$ is given in our dimensionless variables by

$$
L_{F P} W=-\dot{x} \frac{\partial W}{\partial x}+\frac{1}{2} \frac{\partial V}{\partial x} \frac{\partial W}{\partial \dot{x}}+\beta^{\prime}\left(\frac{\partial(\dot{x} W)}{\partial \dot{x}}+\frac{1}{2} \frac{\partial^{2} W}{\partial \dot{x}^{2}}\right) .
$$

The first two terms on the right-hand side of Eq. (8) comprise the convective or Liouville term describing in the absence of dissipation the undamped streaming motion along the energy trajectories in phase space corresponding to Hamilton's equations. The last term (the diffusion term) represents the interchange of energy (dissipative coupling) with the heat bath.

The periodic solutions of Eqs. (5) or (7) cannot describe escape of the particle from the well because the potential (1) contains only one well with period $2 \pi$. To investigate the process across the multiwell potential generated by Eq. (1) one has to obtain a nonperiodic solution of Eqs. (5) or (7) [11]. In order to obtain a nonperiodic solution of the KleinKramers Eq. (7), one makes the ansatz [11]

$$
W\left(x, \dot{x}, t^{\prime}\right)=\int_{-1 / 2}^{1 / 2} \tilde{W}\left(k, x, \dot{x}, t^{\prime}\right) e^{i k x} d k,
$$

where $\tilde{W}$ is periodic in $x$ with period $2 \pi$ and it is assumed that $k$ is restricted to the first Brillouin zone, $-1 / 2 \leq k$ $\leq 1 / 2$. The periodic function $\tilde{W}$ can then be expanded in a truncated Fourier series in $x$ and in orthogonal Hermite functions in $\dot{x}[11]$. A similar approach may be used in solution of the Langevin Eq. (5).

Following Refs. [11,32], we calculate the dynamic structure factor $\widetilde{S}(k, \omega)$, which is the time Fourier transform of the 
TABLE I. Physical systems modeled by Brownian motion in a tilted periodic potential $V(x)=-\gamma(\cos x$ $+y x)$ and described by the Langevin Eq. (5).

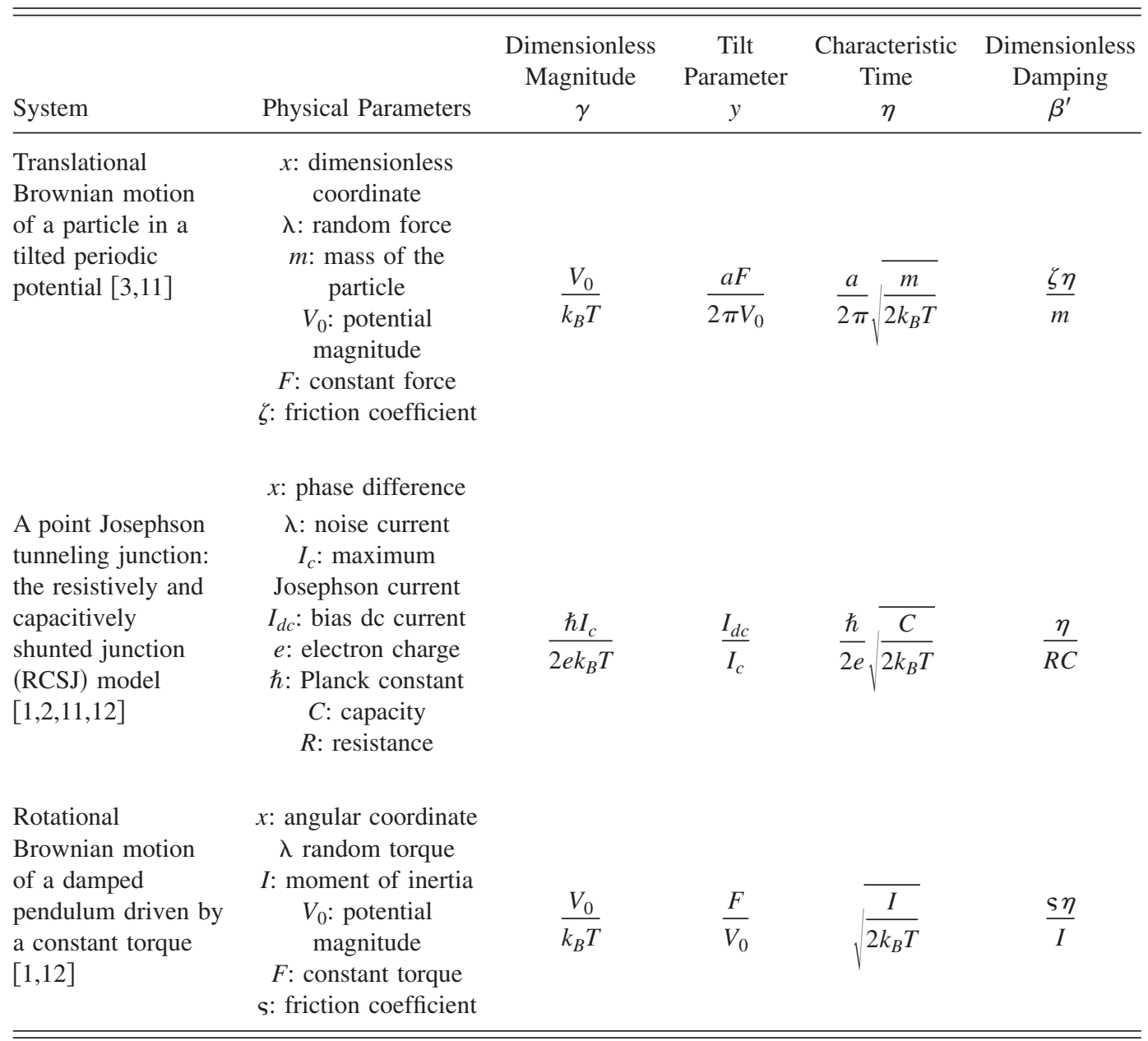

equilibrium translational correlation function $S\left(k, t^{\prime}\right)$ $=\left\langle e^{i k\left[x\left(t^{\prime}\right)-x(0)\right]}\right\rangle_{0}$, where $x(0)$ is the initial value of $x\left(t^{\prime}\right)$, and the angular brackets denotes a stationary averaging. Thus we introduce the set of stationary correlation functions $c_{n, p}\left(k, t^{\prime}\right)$ defined as

$$
c_{n, p}\left(k, t^{\prime}\right)=\frac{1}{\sqrt{2^{n} n !}}\left\langle e^{i k\left[x\left(t^{\prime}\right)-x(0)\right]} e^{i p x\left(t^{\prime}\right)+V\left[x\left(t^{\prime}\right)\right] / 2} H_{n}\left[\dot{x}\left(t^{\prime}\right)\right]\right\rangle_{0},
$$

for the correlation functions $c_{n, p}\left(k, t^{\prime}\right)$. This is accomplished as follows. Recalling that the usual rules of analysis apply to stochastic differential equations of the Stratonovich type $[11,12]$ and noting that $[41]$

$$
\frac{d}{d z} H_{n}(z)=2 n H_{n-1}(z), \quad H_{n+1}(z)=2 z H_{n}(z)-2 n H_{n-1}(z),
$$

where $H_{n}(z)$ is the Hermite polynomial of order $n$ [41]. Equation (5) now may be recast as a hierarchy of equations

one has

$$
\begin{aligned}
\frac{d}{d t^{\prime}}\left\{e^{i(p+k) x\left(t^{\prime}\right)+V\left[x\left(t^{\prime}\right)\right] / 2} H_{n}\left[\dot{x}\left(t^{\prime}\right)\right]\right\}= & \left\{2 n \ddot{x}\left(t^{\prime}\right) H_{n-1}\left[\dot{x}\left(t^{\prime}\right)\right]+\left[i(p+k)+\partial_{x} V\left[x\left(t^{\prime}\right)\right] / 2\right] \dot{x}\left(t^{\prime}\right) H_{n}\left[\dot{x}\left(t^{\prime}\right)\right]\right\} e^{i(p+k) x\left(t^{\prime}\right)+V\left[x\left(t^{\prime}\right)\right] / 2} \\
= & \left\{n\left(2 \lambda\left(t^{\prime}\right) H_{n-1}\left[\dot{x}\left(t^{\prime}\right)\right]-2 \beta^{\prime}(n-1) H_{n-2}\left[\dot{x}\left(t^{\prime}\right)\right]-\beta^{\prime} H_{n}\left[\dot{x}\left(t^{\prime}\right)\right]-H_{n-1}\left[\dot{x}\left(t^{\prime}\right)\right] \partial_{x} V\left[x\left(t^{\prime}\right)\right]\right)\right. \\
& \left.+\left(i(p+k)+\partial_{x} V\left[x\left(t^{\prime}\right)\right] / 2\right)\left(n H_{n-1}\left[\dot{x}\left(t^{\prime}\right)\right]+H_{n+1}\left[\dot{x}\left(t^{\prime}\right)\right] / 2\right)\right\} e^{i(p+k) x\left(t^{\prime}\right)+V\left[x\left(t^{\prime}\right)\right] / 2} .
\end{aligned}
$$

By averaging Eq. (12) over the realizations of $x\left(t^{\prime}\right)$, using the identity [12] 


$$
\overline{F\left[x\left(t^{\prime}\right)\right] H_{n-1}\left[\dot{x}\left(t^{\prime}\right)\right] \lambda\left(t^{\prime}\right)}=\beta^{\prime}(n-1) F(x) H_{n-2}(\dot{x})
$$

(which is valid for an arbitrary function $F\left[x\left(t^{\prime}\right)\right]$ ), and forming the stationary averages $c_{n, q}(t)$ (as described in Ref. [12]), we have

$$
\begin{aligned}
\frac{d}{d t^{\prime}} c_{n, p}\left(k, t^{\prime}\right) & =-n \beta^{\prime} c_{n, p}\left(k, t^{\prime}\right)+\sqrt{\frac{n+1}{2}}\left\{\left[i(p+k)-\frac{\gamma y}{2}\right] c_{n+1, p}\left(k, t^{\prime}\right)-\frac{i \gamma}{4}\left[c_{n+1, p+1}\left(k, t^{\prime}\right)-c_{n+1, p-1}\left(k, t^{\prime}\right)\right]\right\} \\
& +\sqrt{\frac{n}{2}}\left\{\left[i(p+k)+\frac{\gamma y}{2}\right] c_{n-1, p}\left(k, t^{\prime}\right)+\frac{i \gamma}{4}\left[c_{n-1, p+1}\left(k, t^{\prime}\right)-c_{n-1, p-1}\left(k, t^{\prime}\right)\right]\right\} .
\end{aligned}
$$

Having determined the $c_{0, p}(k, t)$ from Eq. (14), the function $S\left(k, t^{\prime}\right)$ can be evaluated as

$$
S\left(k, t^{\prime}\right)=\sum_{p=0}^{\infty} a_{p} c_{0, p}(k, t)=(2 \pi)^{-1} Z \sum_{p=0}^{\infty} c_{0, p}^{*}(k, 0) c_{0, p}(k, t),
$$

where the initial conditions $c_{0, p}(k, 0)$ for $c_{0, p}\left(k, t^{\prime}\right)$ are given by [11]

$$
c_{0, p}(k, 0)=Z^{-1} \int_{0}^{2 \pi} e^{i p x-V(x) / 2} d x, \quad Z=\int_{0}^{2 \pi} e^{-V(x)} d x
$$

(the asterisk denotes the complex conjugate). Here we have noted that

$$
\begin{gathered}
e^{i k x\left(t^{\prime}\right)}=e^{V\left[x\left(t^{\prime}\right)\right] / 2} \sum_{p=0}^{\infty} a_{p} e^{i(p+k) x\left(t^{\prime}\right)}, \\
a_{p}=(2 \pi)^{-1} \int_{0}^{2 \pi} d x e^{-i p x-V(x) / 2}=(2 \pi)^{-1} Z c_{0, p}^{*}(k, 0) .
\end{gathered}
$$

Equation (14) (equivalent to that derived by Risken [11] from the Fokker-Planck equation) is the desired recurrence equation for the statistical moments. This equation can be solved by the matrix continued fraction method to yield the one-sided Fourier transform of the dynamic structure factor $\widetilde{S}(k, \omega)=\int_{0}^{\infty} S\left(k, t^{\prime}\right) e^{-i \omega \eta t^{\prime}} d t^{\prime}$ (see Appendix A). Having determined $\widetilde{S}(k, \omega)$, the longest relaxation time $\tau$ and decay rate $\Gamma \approx \tau^{-1}$ can be evaluated as follows. Defining the decay rate $\Gamma \approx \tau^{-1}$ as

$$
\tau^{-1}=\int_{-1 / 2}^{1 / 2} \tau^{-1}(k) d k
$$

where the time $\tau(k)$ is a characteristic time associated with the long time behavior of the function $S\left(k, t^{\prime}\right)$ which can be approximated as $t \rightarrow \infty$ by an exponential $S\left(k, t^{\prime}\right)$ $\sim h(k) e^{-t / \tau(k)}$ [32]. In the frequency domain as $\omega \rightarrow 0$, this approximation corresponds to

$$
\widetilde{S}(k, \omega)=\frac{h(k)}{i \omega+\tau^{-1}(k)},
$$

where $\tau^{-1}(k)$ can be extracted as [32]

$$
\tau^{-1}(k)=\lim _{\omega \rightarrow 0} i \omega\left[\frac{\tilde{S}(k, 0)}{\widetilde{S}(k, \omega)}-1\right]^{-1} .
$$

The dynamic structure factor $\widetilde{S}(k, \omega)$ can be calculated exactly by solving the differential recurrence Eq. (14) using the matrix continued fractions [11] (as described in Appendix A) and may be compared with the characteristic time $\tau(k)$ and the decay rate $\Gamma \approx \tau^{-1}$ evaluated from Eqs. (19) and (17).

\section{MEL'NIKOV'S UNIVERSAL EQUATION}

An analytical approximation to the decay rate $\tau^{-1}$ in a tilted periodic potential has been obtained by Mel'nikov [17] by reducing the Klein-Kramers equation to an integral equation of Wiener-Hopf type. The Mel'nikov expression for the longest relaxation time $\tau$ is [17]

$$
\tau_{M}=\tau_{I H D} / A\left(\beta^{\prime} \delta, \gamma y\right),
$$

where $\tau_{I H D}$ is the longest relaxation time in the IHD limit which is given by the Kramers IHD formula [17]

$$
\tau_{I H D}=4 \pi \eta\left(\sqrt{\beta^{\prime 2}+2 \gamma}-\beta^{\prime}\right)^{-1} e^{\Delta V},
$$

$\Delta V$ is the height of the lowest barrier (see Fig. 1), viz.,

$$
\Delta V=2 \gamma\left(\sqrt{1-y^{2}}+y \arcsin y-\pi y / 2\right) .
$$

The function $A$ in Eq. (20) is defined as [17]

$$
A(d, g)=\int_{-1 / 2}^{1 / 2} w(k, d, g) d k
$$

where

$$
w(k, d, g)=4 \sin (\pi k) \sin [\pi(k+i g)] e^{-\pi g+\Xi(k, d, g)-\Xi(0,2 d, 0) / 2},
$$




$$
\begin{aligned}
\Xi(k, d, g)= & \sum_{n=1}^{\infty} n^{-1} \cos [\pi n(2 k+i g)] \\
& \times\left\{\operatorname{erfc}\left[\sqrt{n d}\left(\frac{1}{2}+\frac{\pi g}{d}\right)\right] e^{n \pi g}\right. \\
& \left.+\operatorname{erfc}\left[\sqrt{n d}\left(\frac{1}{2}-\frac{\pi g}{d}\right)\right] e^{-n \pi g}\right\},
\end{aligned}
$$

and $\operatorname{erfc}(x)$ is the complementary error function defined as $\operatorname{erfc}(x)=\frac{2}{\sqrt{\pi}} \int_{x}^{\infty} e^{-t^{2}} d t$ [41]. (Other forms of the function $A$ are given in Ref. [17]). For zero tilt $(y=0)$, Eq. (24) can be simplified to yield [32]

$$
w(k, d, 0)=4 \sin ^{2}(\pi k) e^{\Xi(k, d, 0)-\Xi(0,2 d, 0) / 2},
$$

where $\Xi(k, d, 0)=2 \sum_{n=1}^{\infty} n^{-1} \operatorname{erfc}(\sqrt{n d} / 2) \cos (2 \pi n k)$. The parameter $\delta$ is the dimensionless action associated with the path along the top of lowest barrier given by

$$
\begin{aligned}
\delta & =2 \int_{x_{0}}^{-\arcsin y} \sqrt{-V(x)} d x \\
& =2 \sqrt{\gamma} \int_{x_{0}}^{-\arcsin y} \sqrt{\sqrt{1-y^{2}}-\cos x+y(x+\arcsin y)} d x,
\end{aligned}
$$

where $x_{0}$ is an appropriate solution of the equation $V\left(x_{0}\right)=0$. On this path, a particle starts with zero velocity at the top of the barrier and, having descended into the well, returns again to the top of the barrier [36]. For $y \ll 1, \delta$ has the following behavior:

$$
\frac{\delta}{\delta_{0}}=1-\frac{1}{32}\left\{\pi[9-4 \log (\pi y / 4)] y+\sqrt{\pi} y^{3 / 2}\right\}+O\left(y^{2}\right),
$$

where $\delta_{0}=8 \sqrt{2 \gamma}$ is the action for zero tilt. If $y$ approaches 1 , $\delta$ can be approximated as [36]

$$
\delta \approx 3 \delta_{0}[2(1-y)]^{5 / 4} / 10 \text {. }
$$

The normalized action $\delta / \delta_{0}$ evaluated from Eqs. (27)-(29) is shown in Fig. 2. Now $A(d) \rightarrow 1$ at $d \rightarrow \infty$ and $A(d) / d \rightarrow 2$ at $d \rightarrow 0$. Thus for very high damping, $\beta^{\prime} \rightarrow \infty$, Eq. (20) yields the VHD asymptote

$$
\tau_{V H D}=4 \pi \eta \beta^{\prime} \gamma^{-1} e^{\Delta V} .
$$

In the VLD limit $\left(\beta^{\prime} \rightarrow 0\right)$, one has from Eq. (20)

$$
\tau_{V L D}=\frac{\pi \eta}{\beta^{\prime} \delta \sqrt{\gamma / 2}} e^{\Delta V}
$$

which is the result of Büttiker and Landauer [36] (in our notation).

The Mel'nikov method (as other asymptotic methods) is valid in the high barrier (low temperature) limit only. In order to accurately estimate $\tau$ for the low barrier, one should use other methods such as mean first passage time (MFPT) [13]. The MFPT may be easily calculated for all systems with dynamics governed by single variable Fokker-Planck

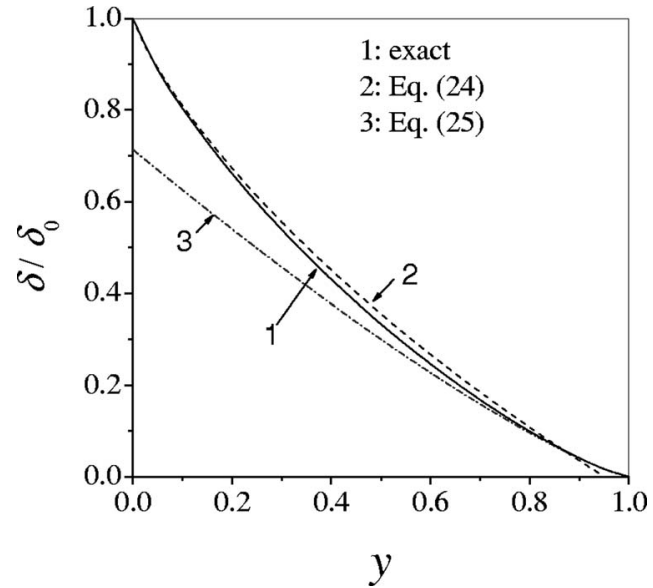

FIG. 2. The normalized action $\delta / \delta_{0}$ vs the tilt parameter $y$. Solid line: Eq. (27). Dashed line: Eq. (28). Dashed-dotted line: Eq. (29).

equations in terms of the equilibrium (stationary) distribution function $W_{0}$ and diffusion coefficient $D^{(2)}$ only. The advantage of such a method is that it allows us to obtain VHD and VLD solutions, valid for all barrier heights including very low barriers, where asymptotic methods (like that of Mel'nikov) are not applicable. In Sec. IV, we apply the theory of MFPT for evaluation of the decay rate $\tau^{-1}$ in the VHD and VLD limits at zero tilt $y=0$. Here the results can be given in a closed form.

\section{THE MFPT ASYMPTOTES FOR THE DECAY RATE AT ZERO TILT}

In the VHD limit $\left(\beta^{\prime} \gg 1\right)$, the appropriate single variable Fokker-Planck (Smoluchowski) equation for the probability density function $W\left(x, t^{\prime}\right)$ is [11]

$$
\frac{\partial}{\partial t^{\prime}} W\left(x, t^{\prime}\right)=\frac{1}{2 \beta^{\prime}}\left\{\frac{\partial}{\partial x}\left[\gamma \sin x W\left(x, t^{\prime}\right)\right]+\frac{\partial^{2}}{\partial x^{2}} W\left(x, t^{\prime}\right)\right\} .
$$

Thus, noting that the diffusion coefficient $D^{(2)}=\left(2 \beta^{\prime}\right)^{-1}$, the longest relaxation time is given by [42]

$$
\begin{aligned}
\tau & \sim \tau_{V H D}^{M F P T}=2 \beta^{\prime} \eta \int_{-\pi}^{\pi} \frac{1}{W_{0}(x)} \int_{-\pi}^{x} W_{0}(\phi) d \phi d x \\
& =2 \beta^{\prime} \eta \int_{-\pi}^{\pi} \int_{-\pi}^{x} e^{\gamma(\cos \phi-\cos x)} d \phi d x,
\end{aligned}
$$

where the equilibrium Boltzmann distribution function $W_{0}(x)$ is given by

$$
W_{0}(x)=\frac{e^{\gamma \cos x}}{2 \pi I_{0}(\gamma)}
$$

and $I_{0}(z)$ is the modified Bessel function [41]. The time $\tau_{V H D}^{M F P T}$ is the time needed for the Brownian particle starting at the top of the barrier $x=-\pi$ to reach, having attended a well bottom, the neighboring top at $x=\pi$.

In the opposite low damping limit $\left(\beta^{\prime} \ll 1\right)$, in order to obtain a single variable Fokker-Planck equation, one may 
introduce as variables the energy of the particle

$$
\varepsilon=\dot{x}^{2}-\gamma \cos x
$$

and the time $w$ (phase) measured along a closed trajectory in phase space. These comprise the action-angle variables [13] of the problem. The energy $\varepsilon$ varies very slowly with time. Consequently, it is a slow variable in comparison to the phase $w$. By using the method of Praestgaard and van Kampen [43], i.e., averaging the Fokker-Planck equation (7) over the fast phase variable $w$, we have a single variable Fokker-Planck equation for the probability density function $W(\varepsilon, t)$ in energy space

$$
\frac{\partial}{\partial t^{\prime}} W\left(\varepsilon, t^{\prime}\right)=2 \beta^{\prime}\left[\frac{\partial}{\partial \varepsilon}\left(\overline{\overline{\dot{x}^{2}}}(\varepsilon)-\frac{1}{2}\right)+\frac{\partial^{2}}{\partial \varepsilon^{2}} \overline{\overline{\dot{x}^{2}}}(\varepsilon)\right] W\left(\varepsilon, t^{\prime}\right),
$$

where the double overbar denotes averaging over the fast phase variable. Noting that the diffusion coefficient is

$$
D^{(2)}=2 \beta^{\prime} \overline{\overline{\dot{x}^{2}}}(\varepsilon)=2 \beta^{\prime}[\varepsilon+\gamma \overline{\overline{\cos x}}(\varepsilon)],
$$

the time $\tau$ is then given by

$$
\tau \sim \tau_{V L D}^{M F P T}=\frac{\eta}{2 \beta^{\prime}} \int_{-\gamma}^{\gamma} \frac{W_{0}^{-1}(\varepsilon) \int_{-\gamma}^{\varepsilon} W_{0}(x) d x}{\varepsilon+\gamma \overline{\overline{\cos x}}(\varepsilon)} d \varepsilon .
$$

The calculation of $W_{0}(\varepsilon)$ and the integrals in Eq. (37) is described in Appendix B. The areas of applicability of the VHD and VLD asymptotes [Eqs. (33) and (37)] are the same as those of the corresponding Fokker-Planck equations (32) and (36), viz., the VHD and VLD limits, respectively. In practice, Eqs. (33) and (37) may be used at $\beta^{\prime}>5$ and $\beta^{\prime}$ $<0.01$.

\section{RESULTS AND DISCUSSION}

The greatest relaxation time predicted by the turnover formula Eq. (20) and the inverse decay rate calculated numerically by matrix continued fraction methods are shown in Figs. 3 and 4 as functions of $\beta^{\prime}$ for different values of the barrier height and tilt parameters. Here, the IHD [Eq. (21)], VHD [Eq. (30)], and VLD [Eq. (31)] asymptotes for $\tau$ are also shown for comparison. Apparently in the high barrier limit, Eq. (20) provides a good approximation to the decay rate for all values of the friction parameter $\beta^{\prime}$ including the VHD, VLD, and the Kramers turnover regions. In spite of very good overall agreement between numerical results and the universal Eq. (20), a marked difference of order of $20 \%$ between numerical and analytical results exists in the VLD region at moderate barriers (this difference decreases with increasing $\gamma$, see Fig. 3). Such a difference has already been noted for other systems (see, e.g., Refs. [22,37]). In order to improve the accuracy of the universal turnover formula in this region, Mel'nikov [44] suggested a systematic way of accounting for finite-barrier corrections. Analysis of the translational Brownian motion in a periodic cosine potential has demonstrated [45] that if such a correction is included,

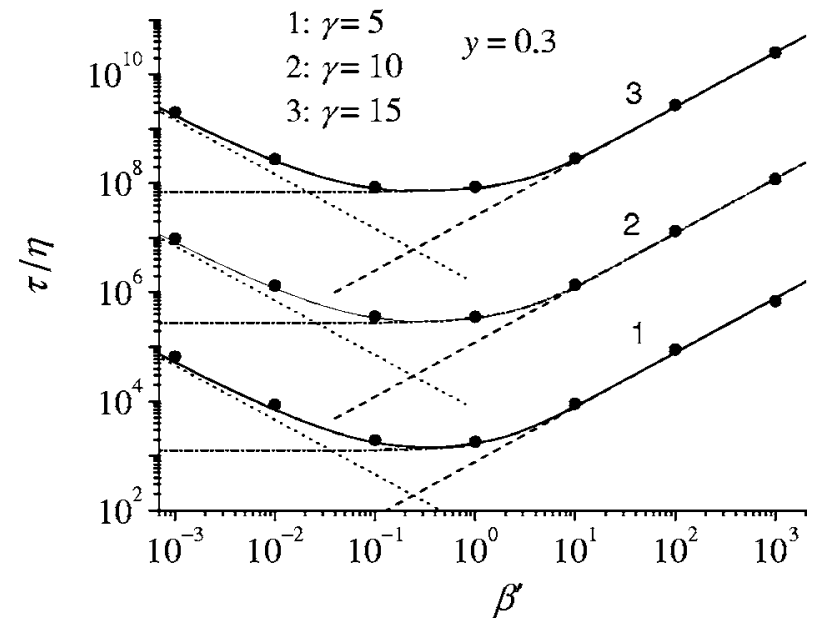

FIG. 3. The longest relaxation time $\tau / \eta$ vs the friction parameter $\beta^{\prime}$ for the tilt parameter $y=0.3$ and different values of the barrier parameter $\gamma=5,10$, and 15. Solid line: the universal Mel'nikov equation (20); dashed line: the VHD equation (30); dashed dotted line: the IHD equation (21); dotted line: the VLD equation (31); filled circles: exact numerical solution, Eq. (17).

the accuracy of the universal formula is considerably improved for Brownian motion in a periodic potential with tilt $y=0$. One would expect a similar improvement for nonzero tilt.

For zero tilt, $y=0$, the greatest relaxation time $\tau$ predicted by the Mel'nikov universal equation (20) and the inverse decay rate calculated numerically by matrix continued fraction methods are shown in Fig. 5 as functions of $\beta^{\prime}$ for different values of the barrier height including the very low barrier $\gamma=0.1$. Here, the VHD [Eq. (33)] and VLD [Eq. (37)] asymptotes for $\tau$ calculated via the theory of MFPT are also shown for comparison. In the VHD and VLD limits, these asymptotes may be used to estimate $\tau$ for all barrier heights. For small barriers (e.g., $\gamma=0.1$ ), the Mel'nikov universal for-

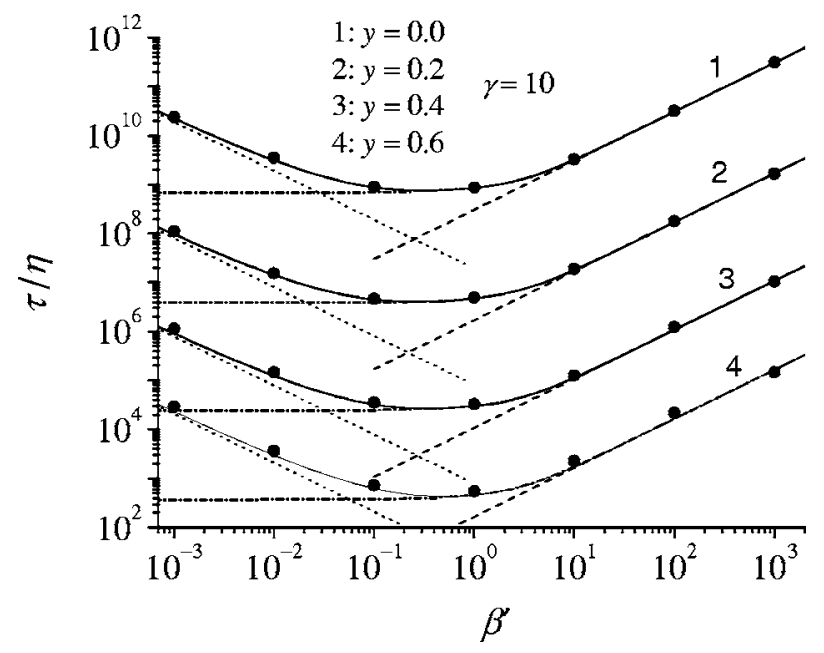

FIG. 4. $\tau / \eta$ vs the friction parameter $\beta^{\prime}$ for the barrier parameter $\gamma=10$ and different values of the tilt parameter $y=0.0,02,04$, and 0.6. Solid line: the universal Mel'nikov equation (20); dashed line: the VHD equation (30); the IHD equation (21); dotted line: the VLD equation (31); filled circles: exact solution, Eq. (17). 


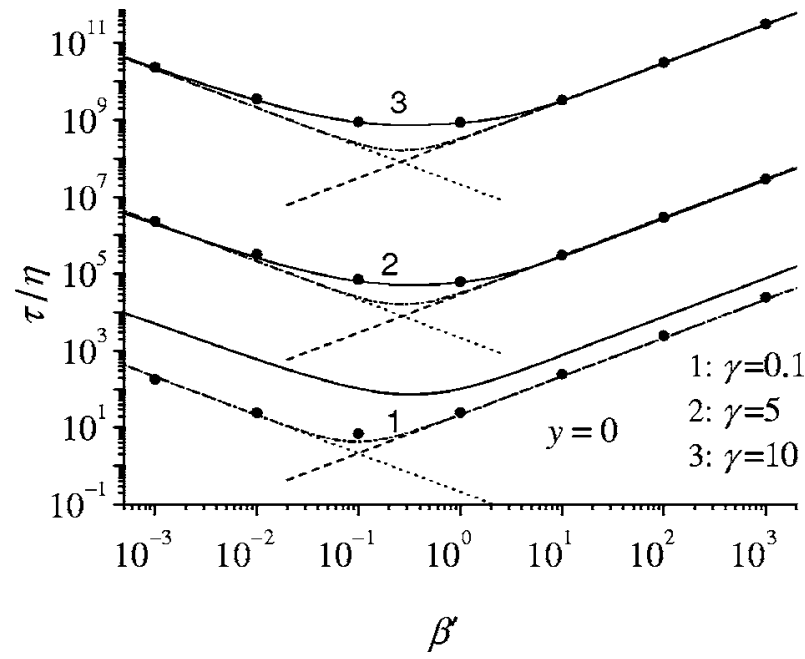

FIG. 5. $\tau / \eta$ vs $\beta^{\prime}$ for the tilt parameter $y=0$ and $\gamma=0.1,5$, and 10. Solid line: the universal Mel'nikov equation (20); dashed line: the VHD MFPT equation (33); dotted line: the VLD MFPT equation (B7); dashed dotted line: the ad hoc extrapolating equation (38); filled circles: exact solution, Eq. (17).

mula is obviously not valid. However, here the simple ad hoc extrapolating equation [13]

$$
\tau \sim \tau_{V H D}^{M F P T}+\tau_{V L D}^{M F P T}
$$

provides a satisfactory estimate of the longest relaxation time $\tau$ for all damping (see Fig. 5).

The real and imaginary parts of the normalized dynamic structure factor $\widetilde{S}(k, \omega) / \widetilde{S}(k, 0)$, for various values of the tilt parameter $y$ are shown in Fig. 6 with barrier parameter $\gamma$ $=10$, the friction coefficient is $\beta^{\prime}=10$, and $k=0.2$. For comparison, we also show in this figure the pure Lorentzian spectra

$$
\frac{\widetilde{S}_{L}(k, \omega)}{\tilde{S}(k, 0)}=\frac{1}{1+i \omega \tau_{k}},
$$

where the relaxation time $\tau_{k}=1 / \operatorname{Re}\left[\tau_{M}^{-1}(k)\right]$ is related to the $\tau_{M}$ from the universal equation (20) via $\tau_{M}^{-1}$ $=2 \int_{0}^{1 / 2} \operatorname{Re}\left[\tau_{M}^{-1}(k)\right] d k$. Apparently the simple equation (39) describes perfectly the low frequency part of the dynamic structure factor $\widetilde{S}(k, \omega) / \widetilde{S}(k, 0)$.

Thus we have demonstrated how the matrix continued fraction solution of nonlinear Langevin equations may be successfully applied to a Brownian particle moving in the tilted periodic potential, Eq. (1), for wide ranges of the barrier parameter $\gamma$, tilt parameter $y$, and the damping parameter $\beta^{\prime}$. We have shown that in the low temperature limit, the Mel'nikov formula for the longest relaxation time, Eq. (20), yields satisfactory agreement with the numerical results for all values of damping. Moreover, the Mel'nikov equation (20) allows one to accurately estimate the damping dependence of the low-frequency parts of the dynamic structure factor $\tilde{S}(k, \omega)$ via the simple approximate analytic formula, Eq. (39). In practical calculations, Eq. (20) may be used for $\gamma \geq 1.5$ and $0 \leq y \leq 0.9$. For $1.0 \geq y>0.9$ (where a parabolic
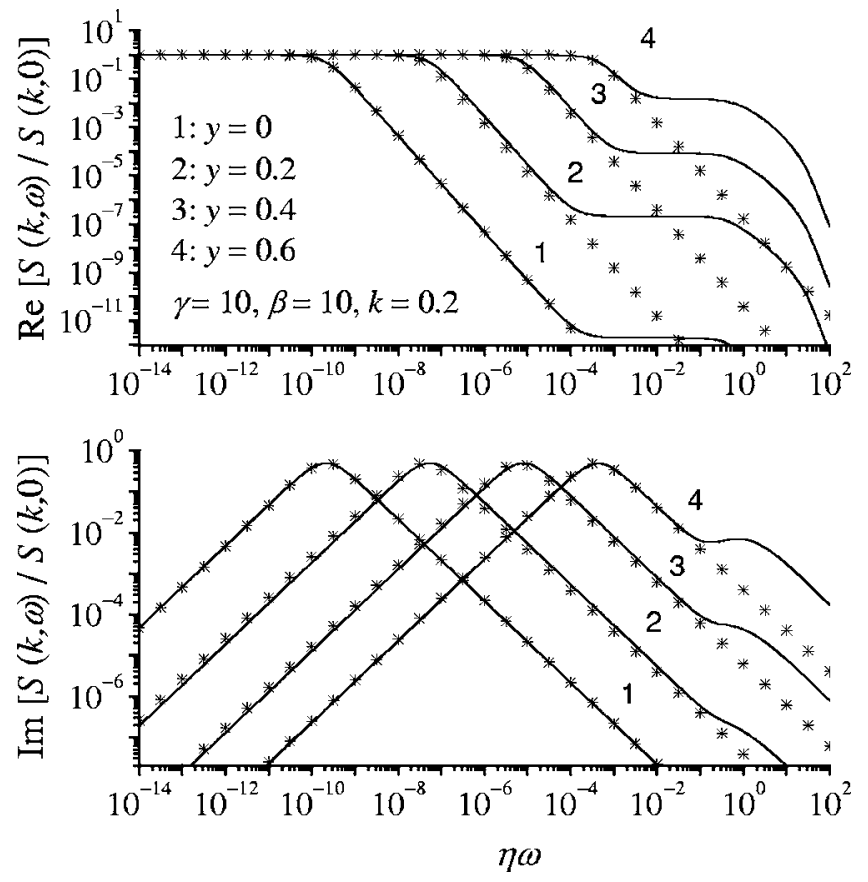

FIG. 6. The real and imaginary parts of the normalized dynamic structure factor $\widetilde{S}(k, \omega) / \widetilde{S}(k, 0)$ vs $\omega \eta$ for various values of the tilt parameter $y$ and for the barrier parameter $\gamma=10$, the friction coefficient $\beta^{\prime}=10$ and $k=0.2$. Solid lines: numerical calculation. Asterisks: Eq. (39).

approximation of the barrier top is no longer valid), the matrix continued fraction solution must be used. For small $\gamma$ (where asymptotic methods like Mel'nikov one are not applicable) and $y=0$, Eqs. (33), (37), and (38) yield a good estimate for the longest relaxation time.

To conclude, we have shown that the universal turnover formula for evaluating the longest relaxation time $\tau$ as a function of the dissipation parameter for Brownian particles in a tilted periodic potential provides in the low-temperature limit excellent agreement with the exact continued fraction solution for all values of the dissipation parameter including the VLD,VHD, and turnover regions. A similar conclusion may be drawn [22-29] for various stochastic systems modeled by Brownian motion in multiwell potentials with equivalent and nonequivalent wells, where the validity of the universal equation for $\tau \sim \Gamma^{-1}$ ( $\Gamma$ is the escape rate) has been verified by comparison with numerical solutions of the underlying Langevin or Fokker-Planck equations. Thus the universal turnover equation for the escape rate appears to yield an effective and powerful tool for evaluating the damping dependence of the prefactor $A$ in Eq. (2) for a wide class of nonlinear stochastic systems even as in the present problem where the stationary solution differs from the MaxwellBoltzmann distribution. It is obvious that the description of the relaxation processes in the context of Eq. (20) neglects quantum effects. These effects are important at very low temperatures and necessitate an appropriate quantum mechanical treatment. As mentioned in Sec. I, Mel'nikov [17] and Rips and Pollak [20] have extended the turnover formula for mechanical particles to account for quantum tunneling in a semiclassical way. We have seen that classical turnover 
formula for the escape rate may be confirmed as an accurate approximation to the exact escape rate of a mechanical Brownian particle because one may exactly solve the corresponding Fokker-Planck equation describing the evolution of the distribution function in phase space using matrix continued fractions. In order to verify formulae for the escape rate which incorporates quantum effects, it is necessary to identify the appropriate quantum mechanical master equation underlying the relaxation process, which becomes the FokkerPlanck equation in the classical limit [46,47]. An appropriate candidate seems to be the Caldeira-Leggett [48] quantum Fokker-Planck equation for the time evolution of the Wigner transform of the reduced density operator (here the relationship between the quantum density operator and the semiclassical distribution function is given by the Wigner transformation [49]). The Caldeira-Leggett approach may be used for all values of damping. In the VHD limit, one can use the quantum Smoluchowski equation, which to leading order coincides with the classical Smoluchowski equation, but contains essential quantum corrections [50-52]. Such an approach to the quantum mechanical problem also lends itself to solution by continued fraction methods $[46,47]$ so that the universal quantum escape rate equations can be tested in a similar manner to that which we have described here.

\section{ACKNOWLEDGMENTS}

This publication has emanated from research conducted with the financial support of Science Foundation Ireland (Project No. 05/RFP/PHY/0070).

\section{APPENDIX A: MATRIX CONTINUED FRACTION SOLUTION} [11]

By introducing the column vectors $\mathbf{C}_{n}\left(k, t^{\prime}\right)$ defined as

$$
\mathbf{C}_{n}\left(k, t^{\prime}\right)=\left(\begin{array}{c}
\vdots \\
c_{n-1,-1}\left(k, t^{\prime}\right) \\
c_{n-1,0}\left(k, t^{\prime}\right) \\
c_{n-1,1}\left(k, t^{\prime}\right) \\
\vdots
\end{array}\right), \quad n=1,2,3, \ldots,
$$

Eq. (14) can be written in the vector tridiagonal form

$$
\begin{aligned}
\frac{d}{d t^{\prime}} \mathbf{C}_{n}\left(k, t^{\prime}\right)= & \mathbf{Q}_{n}^{-}(k) \mathbf{C}_{n-1}\left(k, t^{\prime}\right)-\beta^{\prime}(n-1) \mathbf{C}_{n}\left(k, t^{\prime}\right) \\
& +\mathbf{Q}_{n}^{+}(k) \mathbf{C}_{n+1}\left(k, t^{\prime}\right),
\end{aligned}
$$

where the matrices $\mathbf{Q}_{n}^{+}(k)$ and $\mathbf{Q}_{n}^{-}(k)$ are

$$
\begin{gathered}
\mathbf{Q}_{n}^{+}(k)=i \sqrt{\frac{n}{2}}\left(\begin{array}{ccccc}
\ddots & \vdots & \vdots & \vdots & . \\
\cdots & -1+k+i \gamma y / 2 & -\gamma / 4 & 0 & \cdots \\
\cdots & \gamma / 4 & k+i \gamma y / 2 & -\gamma / 4 & \cdots \\
\cdots & 0 & \gamma / 4 & 1+k+i \gamma y / 2 & \cdots \\
. & \vdots & \vdots & \vdots & \ddots
\end{array}\right), \\
\mathbf{Q}_{n}^{-}(k)=i \sqrt{\frac{n-1}{2}}\left(\begin{array}{ccccc}
\ddots & \vdots & \vdots & \vdots & . \\
\cdots & -1+k-i \gamma y / 2 & \gamma / 4 & 0 & \cdots \\
\cdots & -\gamma / 4 & k-i \gamma y / 2 & \gamma / 4 & \cdots \\
\cdots & 0 & -\gamma / 4 & 1+k-i \gamma y / 2 & \cdots \\
. & \vdots & \vdots & \vdots & \ddots
\end{array}\right) .
\end{gathered}
$$

By Laplacian transformation, Eq. (A1) can be rearranged as the set of matrix three-term recurrence equations

$$
\begin{aligned}
& {\left[\eta s+\beta^{\prime}(n-1)\right] \widetilde{\mathbf{C}}_{n}(k, s)-\mathbf{Q}_{n}^{+}(k) \widetilde{\mathbf{C}}_{n+1}(k, s)-\mathbf{Q}_{n}^{-}(k) \widetilde{\mathbf{C}}_{n-1}(k, s)} \\
& \quad=\delta_{n, 1} \mathbf{C}_{1}(k, 0) .
\end{aligned}
$$

The exact solution of Eq. (A2) for the spectrum $\widetilde{\mathbf{C}}_{1}(k, s)$ is given by in terms of a matrix continued fraction

$$
\widetilde{\mathbf{C}}_{1}(k, s)=\Delta_{1}(k, s) \mathbf{C}_{1}(k, s),
$$

where the matrix continued fraction $\boldsymbol{\Delta}_{n}(k, s)$ is defined by the recurrence equation

$$
\boldsymbol{\Delta}_{n}(k, s)=\left\{\left[\eta s+\beta^{\prime}(n-1)\right] \mathbf{I}-\mathbf{Q}_{n}^{+}(k) \boldsymbol{\Delta}_{n+1}(k, s) \mathbf{Q}_{n+1}^{-}(k)\right\}^{-1}
$$

and $\mathbf{I}$ is the unit matrix. Having determined $\widetilde{\mathbf{C}}_{1}(k, s)$ and noting Eq. (15), one can calculate the dynamic structure factor $\tilde{S}(k, \omega)$ as

$$
\tilde{S}(k, \omega)=(2 \pi)^{-1} Z \mathbf{C}_{1}^{\dagger}(k, 0) \boldsymbol{\Delta}_{1}(k, i \omega) \mathbf{C}_{1}(k, 0),
$$

where the symbol " $\dagger$ " designates transformation of the column vector $\mathbf{C}_{1}(k, 0)$ to a row vector and its conjugation.

The exact matrix continued fraction solution, Eq. (A4), we have obtained is easily computed (algorithms for calculating matrix continued fractions are discussed in Refs. 
$[11,12])$. As far as practical calculations of the infinite matrix continued fraction are concerned, we approximate it by a matrix continued fraction of finite order (by putting $\boldsymbol{\Delta}_{n+1}$ $=\mathbf{0}$ at some $n=N$ ); simultaneously, we confine the dimensions of the infinite matrices $\mathbf{Q}_{n}^{-}, \mathbf{Q}_{n}^{+}$, and $\mathbf{I}$ to a finite value $(2 Q+1) \times(2 Q+1)$. The $N$ and $Q$ were determined in such way that a further increase of $N$ and $Q$ did not change the results. Both $N$ and $Q$ depend on the dimensionless magnitude $(\gamma)$ and damping $\left(\beta^{\prime}\right)$ parameters and must be chosen taking into account the desired degree of accuracy of the calculation. Both $N$ and $Q$ increase with decreasing $\beta^{\prime}$ and increasing $\gamma$.

\section{APPENDIX B: UNDAMPED LIMIT FOR ZERO TILT}

For $\beta^{\prime} \ll 1$, the dynamics of the system differ but little from those of the undamped limit $\left(\beta^{\prime}=0\right)$ when the Langevin force vanishes. For $\beta^{\prime}=0$, the energy of the particle [see Eq. (35)] is a constant of the motion and so the dynamics of the particle in the potential well are described by the following deterministic nonlinear differential equation:

$$
\dot{x}\left(t^{\prime}\right)^{2}=\varepsilon+\gamma \cos x\left(t^{\prime}\right)
$$

or

$$
\frac{d}{d t^{\prime}} \cos x\left(t^{\prime}\right)= \pm \sqrt{\left[\varepsilon+\gamma \cos x\left(t^{\prime}\right)\right]\left[1-\cos ^{2} x\left(t^{\prime}\right)\right]} .
$$

For $-\gamma<\varepsilon<\gamma$, Eq. (B1) has a solution

$$
\cos x\left(t^{\prime}\right)=1-2 m(\varepsilon) \operatorname{sn}^{2}\left(t^{\prime} \sqrt{\gamma / 2} \pm w \mid m(\varepsilon)\right),
$$

where $\operatorname{sn}(u \mid m)$ is the Jacobian doubly periodic elliptic function [41],

$$
\begin{gathered}
w=\int_{0}^{\arcsin \{\sqrt{m(\varepsilon)} \sin [x(0) / 2]\}}\left[1-m(\varepsilon) \sin ^{2} x^{\prime}\right]^{-1 / 2} d x^{\prime}, \\
m(\varepsilon)=\frac{\gamma+\varepsilon}{2 \gamma} .
\end{gathered}
$$

In order to proceed, we recall the Fourier series for $\operatorname{sn}^{2}(u \mid m)$ [53]

$$
m \mathrm{sn}^{2}(u \mid m)=1-\frac{E(m)}{K(m)}-\frac{2 \pi^{2}}{K^{2}(m)} \sum_{n=1}^{\infty} \frac{n q^{n}}{1-q^{2 n}} \cos \left[\frac{n \pi u}{K(m)}\right],
$$

where $K(m)$ and $E(m)$ are the complete elliptic integrals of the first and second kind, respectively [41,53], and $q$ $=\exp [-\pi K(1-m) / K(m)]$. Thus we have from Eqs. (B2), (B3), and (B5)

$$
\overline{\overline{\cos x}}(\varepsilon)=2 \frac{E(m)}{K(m)}-1,
$$

where the double overbar denotes averaging over the fast phase variable, the dependence of the modulus $m$ on $\varepsilon$ is given by Eq. (B4).

In order to evaluate equilibrium averages, one needs an equation for the stationary distribution function $W_{s t}$. On noting that $W_{s t}$ is the equilibrium Maxwell-Boltzmann distribution $W_{0}$, viz.,

$$
\begin{aligned}
W_{0}[ & x(0), \dot{x}(0)] d x(0) d \dot{x}(0) \\
& =\frac{1}{2 \pi^{3 / 2} I_{0}(\gamma)} e^{-\dot{x}^{2}(0)+\gamma \cos x(0)} d x(0) d \dot{x}(0)
\end{aligned}
$$

by making the transformation of the variables $\{x(0), \dot{x}(0)\}$ $\rightarrow\{w, \varepsilon\}$, and by integrating the distribution function $W_{0}(\varepsilon)$ over the phase $w$, we have

$$
W_{0}(\varepsilon) d \varepsilon=\left\{\begin{array}{ll}
\frac{\sqrt{2} K[m(\varepsilon)] e^{-\varepsilon}}{\pi^{3 / 2} I_{0}(\gamma) \sqrt{\gamma}} d \varepsilon \quad(-\gamma<\varepsilon<\gamma) \\
\frac{\sqrt{2} K\left[m^{-1}(\varepsilon)\right] e^{-\varepsilon}}{\pi^{3 / 2} I_{0}(\gamma) \sqrt{\gamma m(\varepsilon)}} d \varepsilon \quad(\varepsilon>\gamma)
\end{array},\right.
$$

with $\int_{-\gamma}^{\infty} W_{0}(\varepsilon) d \varepsilon=1$. Thus according to Eqs. (37) and (B6), in the context of the MFPT approach, $\tau$ in the VLD limit is given by

$$
\frac{\tau}{\eta} \sim \frac{1}{4 \beta^{\prime} \gamma} \int_{-\gamma}^{\gamma} \frac{\int_{-\gamma}^{\varepsilon} e^{\varepsilon-x} K[m(x)] d x}{m(\varepsilon) K[m(\varepsilon)]+E[m(\varepsilon)]} d \varepsilon .
$$

[1] G. Barone and A. Paterno, Physics and Applications of the Josephson Effect (Wiley, New York, 1982).

[2] K. K. Likharev, Dynamics of Josephson Junctions and Circuits (Gordon and Breach, New York, 1986).

[3] W. Dieterich, P. Fulde, and I. Peschel, Adv. Phys. 29, 527 (1980).

[4] W. W. Chow, M. O. Scully, and E. W. Van Stryland, Opt. Commun. 15, 6 (1975).

[5] A. J. Viterbi, Principles of Coherent Communication (McGraw-Hill, New York, 1966).
[6] W. T. Coffey, Adv. Chem. Phys. 63, 69 (1985).

[7] S. G. Chung, Phys. Rev. B 29, 6977 (1984).

[8] W. W. Chow, J. Gea-Banacloche, L. M. Pedrotti, V. Sanders, W. Schleich, and M. O. Scully, Rev. Mod. Phys. 57, 61 (1985).

[9] L. Gammaitoni, P. Hänggi, P. Jung, and F. Marchesoni, Rev. Mod. Phys. 70, 223 (1998).

[10] I. Kh. Kaufman, D. G. Luchinsky, P. V. E. McClintock, S. M. Soskin, and N. D. Stein, Phys. Rev. E 57, 78 (1998).

[11] H. Risken, The Fokker-Planck Equation, 2nd ed. (Springer- 
Verlag, Berlin, 1989).

[12] W. T. Coffey, Yu. P. Kalmykov, and J. T. Waldron, The Langevin Equation, 2nd ed. (World Scientific, Singapore, 2004).

[13] P. Hänggi, P. Talkner, and M. Borkovec, Rev. Mod. Phys. 62, 251 (1990).

[14] H. A. Kramers, Physica (Amsterdam) 7, 284 (1940).

[15] W. T. Coffey, D. A. Garanin, and D. J. McCarthy, Adv. Chem. Phys. 117, 528 (2001).

[16] V. I. Mel'nikov and S. V. Meshkov, J. Chem. Phys. 85, 1018 (1986).

[17] V. I. Mel'nikov, Physica A 130, 606 (1985); Phys. Rep. 209, 1 (1991).

[18] H. Grabert, Phys. Rev. Lett. 61, 1683 (1988).

[19] E. Pollak, H. Grabert, and P. Hänggi, J. Chem. Phys. 91, 4073 (1989).

[20] I. Rips and E. Pollak, Phys. Rev. A 41, 5366 (1990).

[21] M. Topaler and N. Makri, J. Chem. Phys. 101, 7500 (1994).

[22] A. N. Drozdov and P. Talkner, J. Chem. Phys. 109, 2080 (1998).

[23] H. X. Zhou, Chem. Phys. Lett. 164, 285 (1989).

[24] W. T. Coffey, Yu. P. Kalmykov, and S. V. Titov, J. Chem. Phys. 124, 024107 (2006).

[25] R. W. Pastor and A. Szabo, J. Chem. Phys. 97, 5098 (1992).

[26] W. T. Coffey, Yu. P. Kalmykov, and S. V. Titov, J. Chem. Phys. 120, 9199 (2004).

[27] Yu. P. Kalmykov, S. V. Titov, and W. T. Coffey, J. Chem. Phys. 123, 094503 (2005).

[28] P. M. Déjardin, D. S. F. Crothers, W. T. Coffey, and D. J. McCarthy, Phys. Rev. E 63, 021102 (2001).

[29] Yu. P. Kalmykov, J. Appl. Phys. 96, 1138 (2004).

[30] Yu. P. Kalmykov, W. T. Coffey, and S. V. Titov, Fiz. Tverd. Tela (S.-Peterburg) 47, 260 (2005) [Phys. Solid State 47, 272 (2005)].

[31] Yu. P. Kalmykov, W. T. Coffey, B. Ouari, and S. V. Titov, J. Magn. Magn. Mater. 292, 372 (2005).

[32] R. Ferrando, R. Spadacini, and G. E. Tommei, Phys. Rev. E 48, 2437 (1993).

[33] J. D. Cresser, D. Hammonds, W. H. Louisell, P. Meystre, and H. Risken, Phys. Rev. A 25, 2226 (1982).

[34] W. T. Coffey, Yu. P. Kalmykov, and E. S. Massawe, Adv.
Chem. Phys. 85, 667 (1993); W. T. Coffey, Yu. P. Kalmykov, and E. S. Massawe, Phys. Rev. E 48, 77 (1993); W. T. Coffey, Yu. P. Kalmykov, and E. S. Massawe, ibid. 48, 699 (1993); W. T. Coffey, J. L. Déjardin, and Yu. P. Kalmykov, Phys. Rev. E 61, 4599 (2000); Phys. Rev. B 62, 3480 (2000).

[35] M. Büttiker, E. P. Harris, and R. Landauer, Phys. Rev. B 28 , 1268 (1983).

[36] M. Büttiker and R. Landauer, Phys. Rev. B 30, 1551 (1984).

[37] R. Ferrando, R. Spadacini, and G. E. Tommei, Phys. Rev. A 46, R699 (1992).

[38] E. Pollak, J. Bader, B. J. Berne, and P. Talkner, Phys. Rev. Lett. 70, 3299 (1993).

[39] Yu. Georgievskii and E. Pollak, Phys. Rev. E 49, 5098 (1994).

[40] E. Hershkovitz, P. Talkner, E. Pollak, and Yu. Georgievskii, Surf. Sci. 421, 73 (1999).

[41] Handbook of Mathematical Functions, edited by M. Abramowitz and I. Stegun (Dover, New York, 1964).

[42] F. Marchesoni and J. K. Vij, Z. Phys. B: Condens. Matter 58, 187 (1985).

[43] E. Praestgaard and N. G. van Kampen, Mol. Phys. 43, 33 (1981).

[44] V. I. Mel'nikov, Phys. Rev. E 48, 3271 (1993); V. I. Mel'nikov, Phys. Rev. E 50, 627 (1994).

[45] R. Ferrando, R. Spadacini, G. E. Tommei, and V. I. Mel'nikov, Phys. Rev. E 51, R1645 (1995).

[46] J. L. García-Palacios, Europhys. Lett. 65, 735 (2004).

[47] J. L. García-Palacios and D. Zueco, J. Phys. A 37, 10735 (2004).

[48] A. O. Caldeira and A. J. Leggett, Physica A 121, 587 (1983).

[49] E. Wigner, Phys. Rev. 40, 749 (1932).

[50] J. Ankerhold, P. Pechukas, and H. Grabert, Phys. Rev. Lett. 87, 086802 (2001); J. Ankerhold, Chaos 15, 0026106 (2005).

[51] L. Machura, M. Kostur, P. Hänggi, P. Talkner, and J. Łuczka, Phys. Rev. E 70, 031107 (2004).

[52] J. Łuczka, R. Rudnicki, and P. Hanggi, Physica A 350, 60 (2005).

[53] E. T. Whittaker and G. N. Watson, A Course of Modern Analysis, 4th ed. (Cambridge University Press, Cambridge, England, 1927). 